ARTICLE

DOI: $10.1038 / s 41467-018-03752-5$

OPEN

\title{
Rolling up transition metal dichalcogenide nanoscrolls via one drop of ethanol
}

\author{
Xueping Cui ${ }^{1,2}$, Zhizhi Kong ${ }^{1}$, Enlai Gao (1) ${ }^{3}$, Dazhen Huang ${ }^{1,2}$, Yang Hao ${ }^{1,2}$, Hongguang Shen ${ }^{1,2}$, Chong-an Di (1) 1,
} Zhiping $\mathrm{Xu}^{3}$, Jian Zheng ${ }^{1} \&$ Daoben Zhu ${ }^{1}$

Two-dimensional transition metal dichalcogenides (TMDs) have attracted lots of interest because of their potential for electronic and optoelectronic applications. Atomically thin TMD flakes were believed capable to scroll into nanoscrolls (NSs) with distinct properties. However, limited by mechanical strength and chemical stability, production of high-quality TMD NSs remains challenging. Here, we scroll chemical vapor deposition-grown monolayer TMD flakes into high-quality NSs in situ in $5 \mathrm{~s}$ with a nearly $100 \%$ yield by only one droplet of ethanol solution. An obvious photoluminescence is demonstrated in NSs and the self-encapsulated structure makes NSs more insensitive to external factors in optical and electrical properties. Furthermore, based on the internal open topology, NSs hybridized with a variety of functional materials have been fabricated, which is expected to confer TMD NSs with additional properties and functions attractive for potential application.

\footnotetext{
${ }^{1}$ Beijing National Laboratory for Molecular Sciences, Key Laboratory of Organic Solids, Institute of Chemistry, CAS, Beijing 100190, China. ${ }^{2}$ University of Chinese Academy of Sciences, Beijing 100049, China. ${ }^{3}$ Department of Engineering Mechanics and Center for Nano and Micro Mechanics, Applied Mechanics Laboratory, Tsinghua University, Beijing 100084, China. Correspondence and requests for materials should be addressed to

J.Z. (email: zhengjian@iccas.ac.cn)
} 
A tomically two-dimensional (2D) transition metal dichalcogenides (TMDs), such as $\mathrm{MoS}_{2}, \mathrm{MoSe}_{2}, \mathrm{WS}_{2}$, and $\mathrm{WSe}_{2}$, display numerous exceptional electronic and optical properties arising from quantum confinement ${ }^{1,2}$. Complementary to gapless graphene, 2D TMDs with intrinsic band gaps are promising interesting field-effect transistor (FET) and optoelectronic devices. Recently, the main focus of attention has been for the production of their intrinsic or heterojunction structures, their properties, and their applications on $2 \mathrm{D}$ scale $\mathrm{e}^{3-8}$. In addition to changes in $2 \mathrm{D}$ size and form, self-assembly of the atomically thin TMD flakes, as an emerging area, is largely unexplored. Assembly processes by folding and scrolling, i.e., rolling up, can transform relatively simple structures into complex topologies, such as nanoscrolls (NSs), with distinct properties as well as the original excellent characteristics. Indeed, theoretical calculations have predicted the unique topology of $2 \mathrm{D}$ material-based NSs to yield unusual electronic and optical properties $^{9-12}$; thus these NSs have promise as building blocks in flexible electronics, microfluidics, energy storage, self-propelled micromachines, and optical resonators ${ }^{13-18}$. However, experimental realization of such high-quality NSs has only been achieved for graphene $e^{13-16}$ and boron nitride ${ }^{19}$, which exhibited high strength and chemical inertness. Limitations in mechanical strength and chemical stability present difficulties in producing high-quality TMD-NSs ${ }^{20}$. Recently, an argon plasma-assisted method has been demonstrated for the fabrication of amorphous $\mathrm{MoS}_{2}$-NSs, but in this method $\mathrm{MoS}_{2}$ sheets failed to scroll effectively, only edge region of the sheet curved while the large central area remains plane. Moreover, upon argon plasma etching, a serious damage has been brought to the original sheets, where almost half of sulfur atoms were removed. The obtained NSs exhibited low crystallinity even to be amorphous ${ }^{21}$. A highquality TMD-NS is highly desirable for both fundamental studies and potential applications; however, its reliable experimental fabrication still remains challenging.

Here, we show a very simple method for fabricating highquality TMD-NSs, which only requires one droplet of ethanol solution to scroll chemical vapor deposition (CVD)-grown monolayer TMD flakes in situ in $5 \mathrm{~s}$ with a nearly $100 \%$ yield. TMD-NSs are promising optoelectronic materials with potential applications in optoelectronic devices because of the high FET mobility. Owing to self-encapsulated structure, the optical and electrical properties of NSs are more insensitive to external factors, while the electrical performance of $2 \mathrm{D}$ materials flakes varies with both the underlying substrate and the environment. In addition, because of the internal open topology, the interlayer spacing of TMD-NSs can be easily expanded to accommodate a variety of functional materials, including organic small molecules, polymers, nanoparticles, and 2D materials, as well as biological substances. These features are very attractive for applications in solar cells, photodetectors, flexible logic circuits, energy storage, and sensors.

\section{Results}

Rolling up CVD-grown TMD monolayer flakes. In a typical experiment, large-area, monolayer TMD flakes were synthesized on a $\mathrm{SiO}_{2} / \mathrm{Si}$ substrate by CVD. Then, the TMD flakes were scrolled into NSs in just $5 \mathrm{~s}$ with a nearly $100 \%$ yield by placing one drop of ethanol solution (volume ratio of ethanol:water = 2:1) on their surface. Other aqueous solutions, such as methanol, tetrahydrofuran, dimethylformamide, and $N$-methyl-2-pyrrolidone, can also be used for TMD flakes scrolling (Supplementary Fig. 1, 2 and Supplementary Note 1). In addition, the scrolling proceeded well for TMD flakes grown on other substrates, such as $\mathrm{Si}_{3} \mathrm{~N}_{4}$ and sapphire (Supplementary Fig. 3). To clarify the TMDNSs formation process, the entire procedure was recorded using a CCD camera equipped on an optical-microscope (Supplementary Movie 1). A series of steps that might have led to the formation of the TMD-NSs are proposed in Fig. 1. Monolayer TMD flakes were grown on a substrate at a high temperature $\left(\geq 720^{\circ} \mathrm{C}\right)$. During the process of cooling down to room temperature, a strain was introduced to the TMD flakes because of the mismatch in thermal expansion coefficients between the TMD flake and the substrate. Stable TMD flakes were achieved when the strain and substrate adhesion were balanced. When an ethanol solution spread onto the surface of TMD flakes, the liquid film intercalated into the TMD flakes and the substrate, as demonstrated by the contrast color change in the flakes (Supplementary Fig. 4). With the liquid intercalation, part of the TMD flake was first released from the substrate to become freestanding as shown in Fig. 1. Under the built-in strain, the released part of the flake curved out of the plane and continued to roll up to a complete NS in the solution. An energy analysis of the $\mathrm{MoS}_{2}$ flakes before and after scrolling was presented in the Supplementary Note 2. The energy barrier required to bend the freestanding flake out of plane was proved to be relatively low, indicating the easy nucleation of a scroll. In addition, the strain energy induced from the thermal mismatch of the $\mathrm{MoS}_{2}$ flake was found high enough to activate the formation of scrolls after the liquid intercalation and the adhesion energy of the $\mathrm{MoS}_{2}$ flake with substrate decreasing (Supplementary Fig. 5). Strain as the stimulus to the NSs

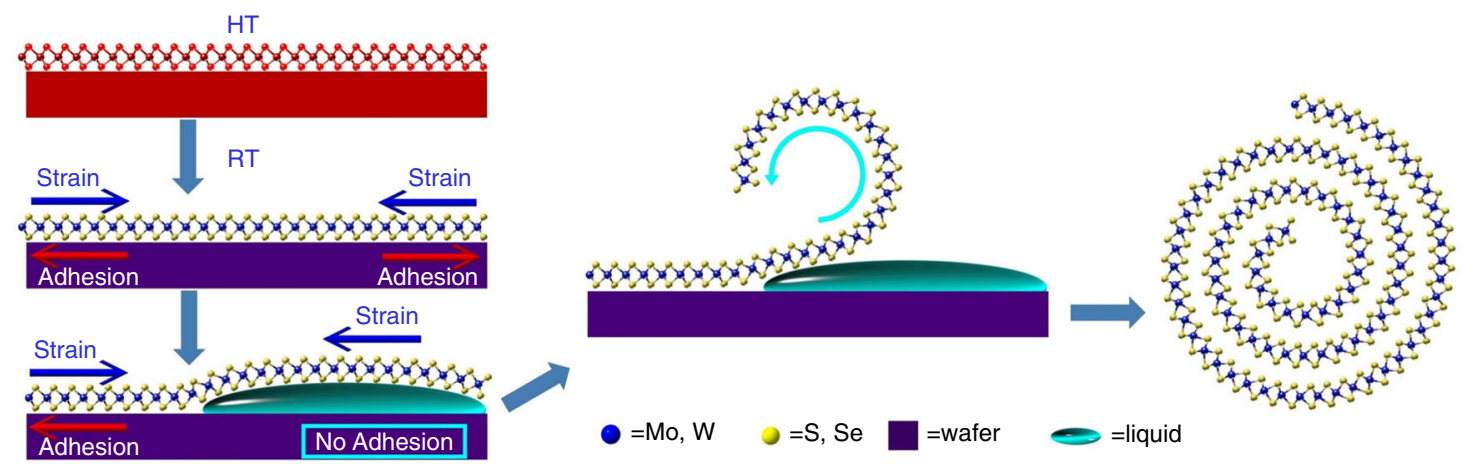

Fig. 1 Schematic of self-scrolling of chemical vapor deposition (CVD)-based transition metal dichalcogenide (TMD) monolayer flakes. A proposed series of steps leading to the formation of TMD-NSs is shown: (I) initial TMD monolayer growth on a substrate at a high temperature ( $\mathrm{HT}$ ) (e.g., $720,825^{\circ} \mathrm{C}$ ); (II) the generation of a strain, balanced with the adhesion from substrate, in the TMD monolayer while cooling down to room temperature (RT); (III) the insertion of liquid between the TMD monolayer and substrate and the disappearance of the adhesion; (IV) the TMD flakes curve out of the plane driven by the strain; $(V)$ the final formation of TMD-NSs 
formation was also suggested by the following fact. When treated with ethanol solution, freshly produced TMD flakes on substrates were more prone to scroll in the shortest time with the highest yield, while the aged samples exhibited slightly decreased scrolling speed and yield. A few cracks were observed in aged samples by scanning electron microscopy (SEM, Supplementary Fig. 6), and these cracks could have partially relieved the strain.

TMD flakes were observed to prefer to scroll along one edge where the adhesion with the substrate was first relaxed upon the intercalation of the liquid. As the edges of CVD-grown TMD single crystals have a zigzag orientation ${ }^{4,22,23}$, the chirality of their NSs are defined (Supplementary Fig. 7). For large-area polycrystalline films without regular edges, the orientation and chirality of their NSs are generally random (Fig. 2a, b). Thus, the controllable scrolling of TMD films was attempted (Fig. 2c-e and Supplementary Fig. 8). Using a focused ion beam (FIB), a $\mathrm{MoS}_{2}$ polycrystalline film was patterned into parallel ribbons with defined width and direction (Fig. 2c) to produce long NS arrays (Fig. 2d). Using FIB etching a second time, long NSs were further arranged into periodic arrays with a controlled length (Fig. 2e). These NS arrays have promise for wide application, such as integrated circuits and matrix displays. We assume that horizontal TMD-NS arrays with controllable chirality could potentially be achieved by controlling the crystal orientation of the ribbon edges via etching from one large-area TMD single crystal, which is prevented by the technique to synthesize largearea TMD single crystals.

Morphology characterization. CVD-grown TMD flakes are high quality even comparable to intrinsic flakes ${ }^{23,24}$. The ethanol solution was clean and harmless; therefore, no defects or impurities were introduced into the high-quality TMD-NSs (Supplementary Fig. 9). Based on this method, several typical TMD-NSs, including those of $\mathrm{MoS}_{2}$ (Fig. $2 \mathrm{f}$ and Supplementary Fig. 10), $\mathrm{WS}_{2}$ (Fig. 2g), $\mathrm{MoSe}_{2}$ (Fig. 2h), and $\mathrm{WSe}_{2}$ (Fig. 2i), were successfully fabricated. Most of the NSs displayed a straight and compact appearance. High-resolution transmission electron microscopy (HR-TEM) was used to further examine the TMD-NSs microstructure, which was found to be multiwalled and tubular (Fig. 2j-m). The diameter and sidewall thickness of the TMD-NS varied with the size of the original flake. The hollow diameters were $10-40 \mathrm{~nm}$. In addition, the high-magnification images of the sidewalls and the selected area electron diffraction (SAED) both showed that the TMD layers in the NSs were stacked uniformly and compactly (Fig. 2j-m inset and Supplementary Fig. 11). The distance between adjacent walls was approximately $6.21 \AA$, $6.41 \AA$, $6.35 \AA$, and $6.45 \AA$ for $\mathrm{MoS}_{2^{-}}, \mathrm{WS}_{2^{-}}, \mathrm{MoSe}_{2^{-}}$, and $\mathrm{WSe}_{2}-\mathrm{NSs}$, respectively, which matched the interlayer spacing in bulk TMDs $\left(6.15 \AA, 6.36 \AA, 6.45 \AA\right.$, and $6.49 \AA$ for $\mathrm{MoS}_{2}, \mathrm{WS}_{2}, \mathrm{MoSe}_{2}$, and
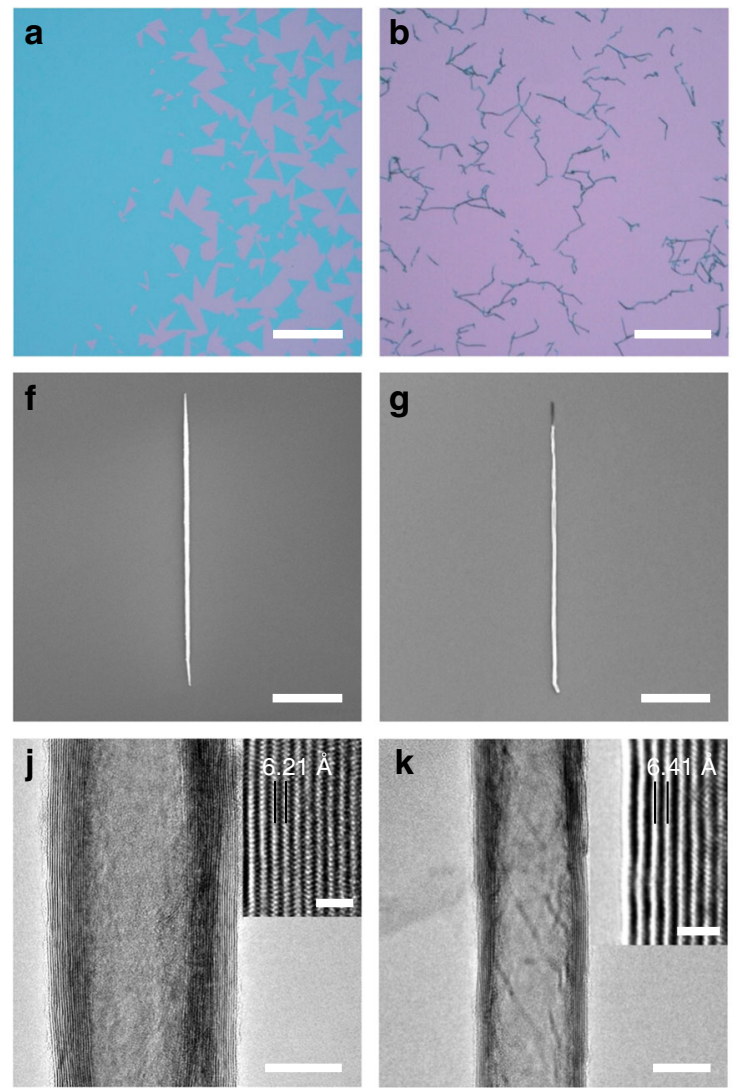
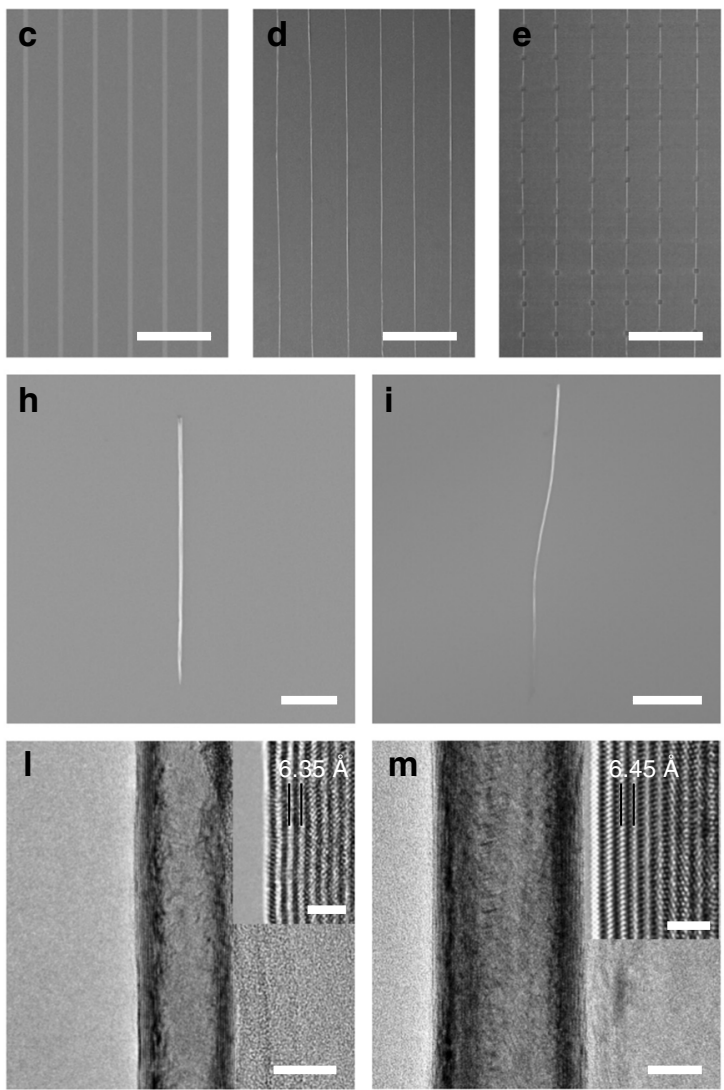

Fig. 2 Transition metal dichalcogenide (TMD)-nanoscrolls (NSs) from self-scrolling chemical vapor deposition (CVD)-based TMD monolayer flakes. a Optical image of CVD-grown $\mathrm{MoS}_{2}$ monolayer flakes on a $\mathrm{SiO}_{2} / \mathrm{Si}$ substrate (the purple area represents the substrate, the green area represents the $\mathrm{MoS}_{2}$ monolayers; scale bar, $500 \mu \mathrm{m}$ ). b Optical image of $\mathrm{MoS}_{2}-\mathrm{NSs}$ on a $\mathrm{SiO}_{2} / \mathrm{Si}$ substrate (scale bar, $100 \mu \mathrm{m}$ ). c-e The fabrication process of a MoS $\mathrm{S}_{2}-\mathrm{NS}_{\text {array }}$ (scanning electron microscopy-SEM image shown, scale bars, $50 \mu \mathrm{m}$ ). c Large-area MoS $_{2}$ monolayer film patterned into ribbons by focused ion beam (FIB) etching (the dark region represents $\mathrm{MoS}_{2}$ film, the bright area is bare substrate). d Long MoS 2 -NSs made from the controllable scrolling of the

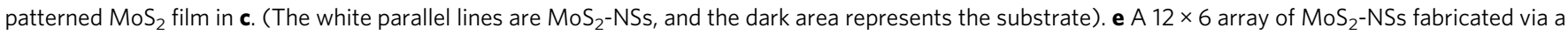

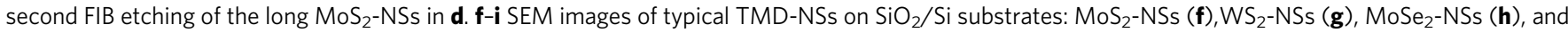

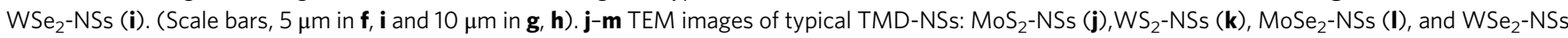
(m). (Scale bars, $20 \mathrm{~nm}$ ). Inset: High-magnification images of sidewalls of TMD-NSs (scale bars, $2 \mathrm{~nm}$ ) 
a
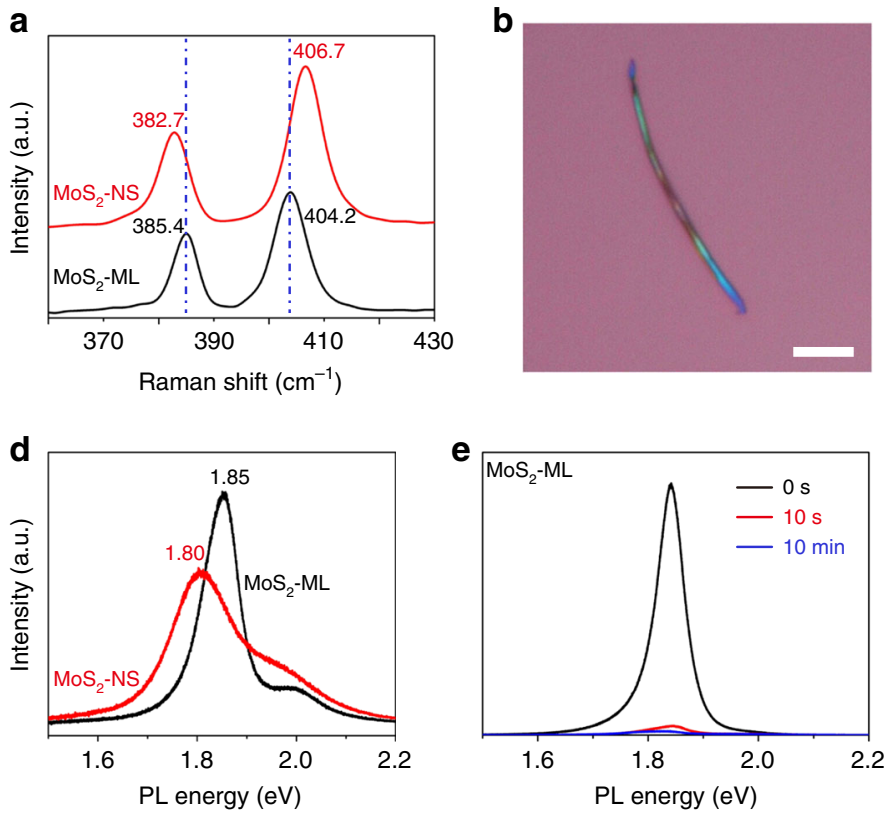
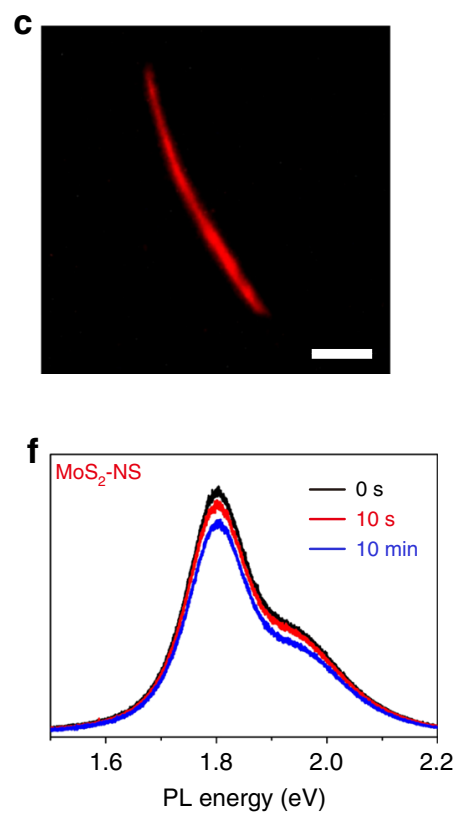

Fig. 3 Optical characterization of $\mathrm{MoS}_{2}-\mathrm{NSs}$ (nanoscrolls). a Raman spectra of chemical vapor deposition (CVD)-grown $\mathrm{MoS}_{2} \mathrm{monolayers}\left(\mathrm{MoS} \mathrm{S}_{2}-\mathrm{ML}\right.$, black line) and $\mathrm{MoS}_{2}-\mathrm{NSs}$ (red line) with $532 \mathrm{~nm}$ excitation. $\mathbf{b}$ Optical image of a typical $\mathrm{MoS}_{2}-\mathrm{NS}$. c Corresponding fluorescence image of the MoS $\mathrm{NS}_{2}$ in b. The fluorescence image was obtained with an excitation wavelength of 510-560 nm. d PL spectra from MoS $_{2}$ monolayer (black line) and MoS ${ }_{2}-\mathrm{NSs}$ (red line) (532 nm excitation). e, f PL spectra response of $\mathrm{MoS}_{2}$ monolayer (e) and $\mathrm{MoS}_{2}-\mathrm{NSs}$ (f) with $\mathrm{NH}_{3}$ doping for $\mathrm{O}$ s (black line), $10 \mathrm{~s}$ (red line), and 10 min (blue line) (scale bars, $5 \mu \mathrm{m}$ in $\mathbf{b}$ and $\mathbf{c}$ )

$\mathrm{WSe}_{2}$, respectively), indicating both the tight scrolling of the TMD flakes and the lack of interlayer contamination.

Optical characterization. Raman spectra of the TMD-NSs were recorded using $532 \mathrm{~nm}$ excitation (Fig. 3a and Supplementary Fig. 12a-c). The Raman signature of monolayer $\mathrm{MoS}_{2}$ flakes is consistent with that of mechanically exfoliated monolayer $\mathrm{MoS}_{2}{ }^{25}$. A similar Raman signature was also found for $\mathrm{MoS}_{2}-\mathrm{NSs}$, demonstrating the good crystallinity of the $\mathrm{MoS}_{2}-\mathrm{NSs}$. A blue shift was observed in the $\mathrm{A}_{1 \mathrm{~g}}$ mode from $404.2 \mathrm{~cm}^{-1}$ for the monolayer $\mathrm{MoS}_{2}$ to $406.7 \mathrm{~cm}^{-1}$ for the $\mathrm{MoS}_{2}-\mathrm{NSs}$, and this shift was attributed to the van der Waals interactions between neighboring NS layers ${ }^{25,26}$. Additionally, a red shift in the $\mathrm{E}_{2 \mathrm{~g}}^{1}$ mode was detected from $385.4 \mathrm{~cm}^{-1}$ for the monolayer $\mathrm{MoS}_{2}$ to $382.7 \mathrm{~cm}^{-1}$ for the $\mathrm{MoS}_{2}-\mathrm{NSs}$, resulting from stacking-induced structural changes, long-range Coulombic interlayer interactions ${ }^{25,27}$, or/and a slight tension owing to the bending deformation of the flake ${ }^{28}$. The frequency shift behavior reflects structural changes after scrolling, and the changes were also observed for $\mathrm{WS}_{2^{-}}, \mathrm{MoSe}_{2^{-}}$, and $\mathrm{WSe}_{2^{-}}$ NSs (Supplementary Fig. 12a-c).

Semiconducting TMD monolayers are direct-bandgap materials because of quantum confinement effects ${ }^{29}$. The strong photoluminescence (PL) and a wide range of band gaps of the monolayer TMD semiconductors indicate their potential for use in $2 \mathrm{D}$ optoelectronics, such as optical energy conversion devices ${ }^{30}$ or photodetectors $^{31}$. Despite having a stacking structure similar to that of bulk TMDs, these TMD-NSs were shown to have an obvious PL, in contrast with the bulk counterparts, in which PL is negligible ${ }^{29}$. Red luminescence from a typical $\mathrm{MoS}_{2}$-NS was clearly observed by fluorescence imaging, indicating that the PL was sufficiently strong to be imaged (Fig. 3b, c). In the PL spectra of Fig. 3d, A, B direct excitonic transitions ${ }^{29}$ from $\mathrm{MoS}_{2}-\mathrm{NSs}$ appeared at around $1.80 \mathrm{eV}$ $(688 \mathrm{~nm})$ and $1.95 \mathrm{eV}(635 \mathrm{~nm})$, matching their absorption resonances (Supplementary Fig. 13). Compared with monolayer $\mathrm{MoS}_{2}$, a red shift in the A exciton transition, approximately of
30-60 meV was found for $\mathrm{MoS}_{2}$-NSs and was accompanied by an increase in the full-width at half-maximum of $50-80 \mathrm{meV}$. The red shift of A peak, an indicator of the variation of the electronic structure, can be explained by the stacking and tension effects in the $\mathrm{NSs}^{29,32,33}$, while PL peak broadening is presumably owing to the overlap of signals from several $\mathrm{MoS}_{2}$ layers in a NS. Analogous PL behavior was also observed for the $\mathrm{WS}_{2^{-}}, \mathrm{MoSe}_{2^{-}}$, and $\mathrm{WSe}_{2}-\mathrm{NSs}$ (Supplementary Fig. 12d-f).

It is well known that monolayer TMD atoms are fully exposed, which makes their optical and electrical properties sensitive to environmental factors. As shown in Fig. 3e, f, when exposed to $\mathrm{NH}_{3}$, the PL intensity of monolayer $\mathrm{MoS}_{2}$ flakes was quenched rapidly and drastically; in contrast, the PL intensity of $\mathrm{MoS}_{2}-\mathrm{NSs}$ did not show a pronounced decrease. Apparently, the optical properties of $\mathrm{MoS}_{2}$-NSs were less dependent on the environment, which might be attributed to the self-encapsulated structure, where most of the $\mathrm{MoS}_{2}$ flakes were rolled up inside and sealed by the outermost layers.

FET performance. Since TMD-NSs are semiconductors, and there are no previous reports on their FET properties, it is interesting to explore this issue here. Device characterization is easy for $\mathrm{MoS}_{2}$-NSs because the NSs have been produced on a $\mathrm{SiO}_{2} / \mathrm{Si}$ substrate. Defining source and drain electrodes were directly deposited on the top of $\mathrm{MoS}_{2}$-NSs via a shadow mask. This process avoided organic impurity contamination that might occur during traditional electron-beam lithography. The FETs based on the $\mathrm{MoS}_{2}-\mathrm{NS}$ s exhibited good FET behavior, and the current/voltage characteristics conformed well to conventional transistor models in both saturated and linear regimes in $\mathrm{N}_{2}$ (Fig. 4 and Supplementary Fig. 14). According to the equation below, we calculated the mobility in the saturation regime.

$$
I_{\mathrm{D}}=(W / 2 L) C_{\mathrm{i}} \mu\left(V_{\mathrm{G}}-V_{\mathrm{T}}\right)^{2},
$$


a
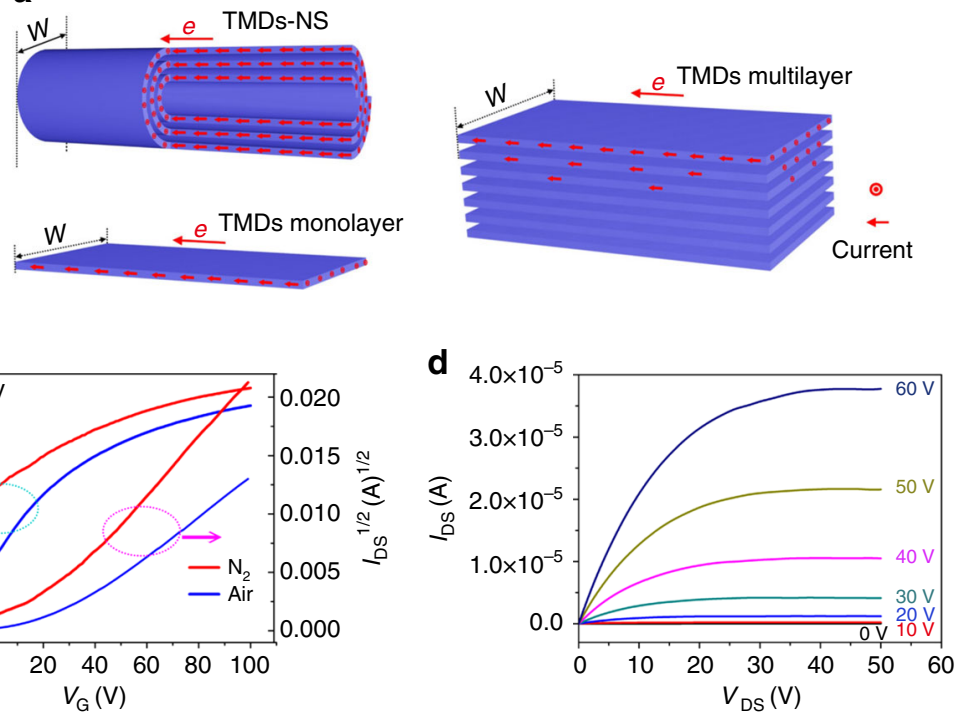

b
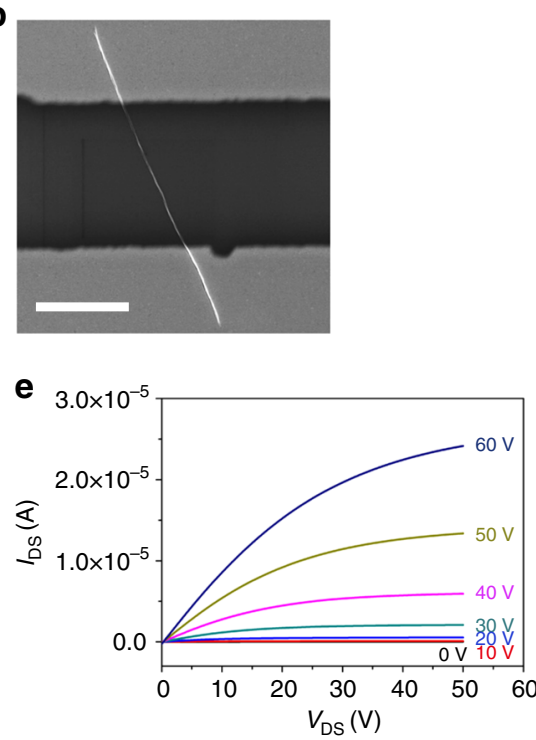

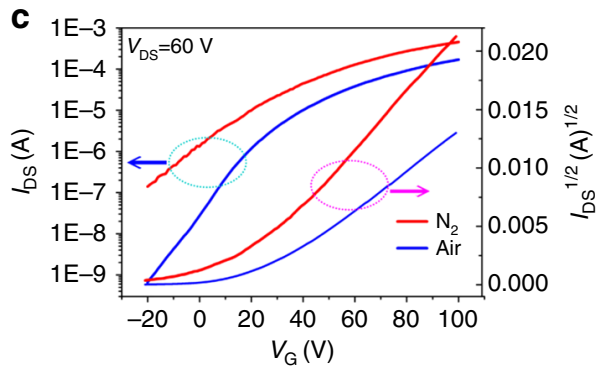

Fig. 4 Electrical characteristics of $\mathrm{MoS}_{2}-\mathrm{NSs}$ (nanoscrolls). a Schematic representations of current conduction in transition metal dichalcogenide (TMD)nanoscrolls (NSs), TMDs monolayer, and TMDs multilayer under bias. The $W$ represents the conduction channel width. The NSs made from the below TMDs monolayer have a much shorter $W$. For TMDs multilayer, the current only passes through the outermost shell layers. $\mathbf{b}$ SEM image of a typical $\mathrm{MoS}_{2}$-NS FET (scale bar, $10 \mu \mathrm{m}$ ). c Transfer characteristics of $\mathrm{MoS}_{2}-\mathrm{NS}$ tested in $\mathrm{N}_{2}$ (red) and air (blue). d Output characteristics of MoS $\mathrm{S}_{2}-\mathrm{NS}$ tested in $\mathrm{N}_{2}$. e Output characteristics of $\mathrm{MoS}_{2}-\mathrm{NS}$ tested in air

where $L$ and $W$ are the channel length and width, respectively, $C_{\mathrm{i}}$ is the insulator capacitance per unit area $\left(11 \mathrm{nF} \mathrm{cm}{ }^{-2}\right), \mu$ is the FET mobility, and $V_{\mathrm{T}}$ is the extrapolated threshold voltage. More than 300 devices were tested. The electron mobility of NSs was measured to be mostly in the range $200-700 \mathrm{~cm}^{2} \mathrm{~V}^{-1} \mathrm{~s}^{-1}$, with an on/off ratio of over $1.0 \times 10^{5}$. The mobility of $\mathrm{MoS}_{2}$-NSs, nearly 30 -fold greater than that of monolayer $\mathrm{MoS}_{2}$ flakes (10-20 $\left.\mathrm{cm}^{2} \mathrm{~V}^{-1} \mathrm{~s}^{-1}\right)^{34}$, should be ascribed to the high current density for compact scroll topology. Invoking an Archimedean spiral model, we quantified the change in the conduction channel widths upon scroll (the detailed analysis is provided in Supplementary Note 3). A considerable reduction in conduction channel widths was achieved after scrolling, while there is no significant decline in the current for the flakes upon scrolling process (Supplementary Fig. 15, 16). This led to a higher current density and mobility. In addition, benefiting from the compact scrolling, conductive layers in a NS compactly stacked layer by layer. The adjacent layers acted as ideal substrates for each other with atomically smooth surface, relatively no dangling bonds and charge traps, which also enhanced electron transporting efficiency ${ }^{35}$. With the similar stacked layer structures, however, the $\mathrm{MoS}_{2}$-NSs also exhibited much greater electron mobility than did the multilayer $\mathrm{MoS}_{2}$ flakes ${ }^{36}$. It can be explained that in a $\mathrm{MoS}_{2}$-NS, electrons are transported through the whole scrolled $\mathrm{MoS}_{2}$ flake, while in multilayer $\mathrm{MoS}_{2}$ flakes, electrons are blocked by van der Waals gaps while migrating to inner layers; thus, only a few shell layers contribute to the carriers transmission (Fig. 4a). The monolayer $\mathrm{MoS}_{2}$-based FETs were observed to be very sensitive to atmosphere. However, there was no obvious decrease in FET mobility for the $\mathrm{MoS}_{2}$-NSs when they were tested in air (Fig. 4c-e)

because most of each electrically active $\mathrm{MoS}_{2}$ layers is encapsulated within each NS and the compact stacked (with an interlayer distance of $0.62 \mathrm{~nm}$ ) morphology can efficiently prevent oxygen from intercalating into the $\mathrm{MoS}_{2}$-NSs to deteriorate transistors performance. The high mobility and unique self-encapsulated morphology make $\mathrm{MoS}_{2}$-NSs promising in FETs.
Hybrid TMD-NSs. As mentioned above, TMD-NSs are inert to the outsides owing to their self-encapsulated structures; however, they are inclined to be doped and form composites because of their internal open topological structures. With a pretreatment, TMD flakes can be hybridized with diverse loads, which become sandwiched into the van der Waals gaps upon flakes scrolling (Fig. 5a). In this paper, we produced $\mathrm{MoS}_{2}$-NSs hybridized with gold nanoparticles (AuNPs) (Fig. 5b), graphene oxide (GO) (Fig. 5c), pentacene (Fig. 5d), copper (II) phthalocyanine (CuPc) (Fig. 5e), poly $\left\{2,2^{\prime}-[(2,5-b i s\right.$ (2-hexyldecyl) -3,6-dioxo- -2,3,5,6-tetrahydropyrrolo[3,4-c] pyrrole-1,4-diyl)dithiophene] $-5,5^{\prime}$-diyl-alt-thiophen- -2,5-diyl\} (PDPP3T) (Fig. 5f), DNA (Fig. 5g) and polypeptide (Fig. 5h). In contrast with nanotubes, the lattice spacing of NSs can adjust automatically upon hybridization with foreign substances of different sizes, and this adjustment can be evaluated by HR-TEM. Figure $5 \mathrm{~b}-\mathrm{h}$ displayed the lattice spacing of hybrid NSs, where lattice expansion was observed for all composite NSs, and some composite components, such as AuNPs, were large enough to be directly visualized (Fig. 5b). Moreover, Raman spectroscopy was utilized to verify the presence of foreign substances. As a typical example, the intercalation of GO into NSs was verified by the emergence of the $D, G$, and $2 D$ peaks of $G O$ (around 1350, 1600, $\left.2671 \mathrm{~cm}^{-1}\right)^{37}$, as well as vibrational modes (around $383,406 \mathrm{~cm}^{-1}$ ) for $\mathrm{MoS}_{2}$ (Supplementary Fig. 17a). In addition, TMD-NSs can be conferred with additional properties and functions upon hybridization with other functional materials, such as organic small molecules, polymers, nanoparticles, and 2D materials, as well as biological substances. For example, after hybridization with PDPP3T, a new fluorescence peak appeared at about $1.42 \mathrm{eV}(873 \mathrm{~nm})$ for $\mathrm{MoS}_{2}$-NSs (Supplementary Fig. 17b), indicating that the hybridization broadened $\mathrm{MoS}_{2}$-NS emission spectrum and could eventually extend their optical applications. Furthermore, the intercalated NS by PDPP3T exhibited a good stability in air, due to the self-encapsulated structure (Supplementary Fig. 18). 
a

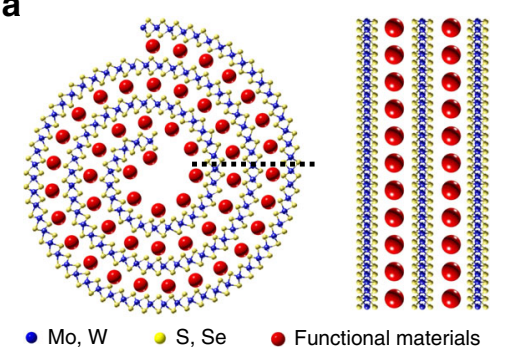

b

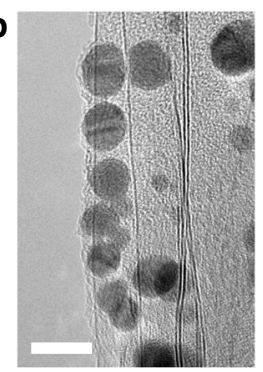

C
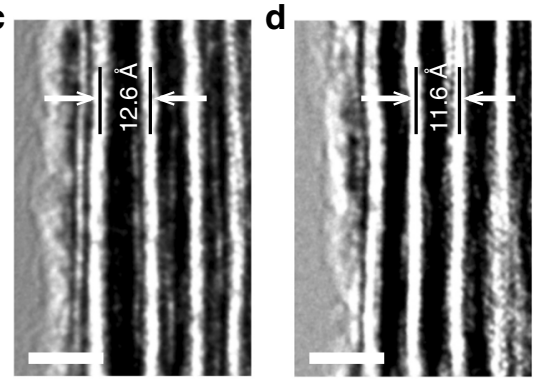

$\mathbf{f}$
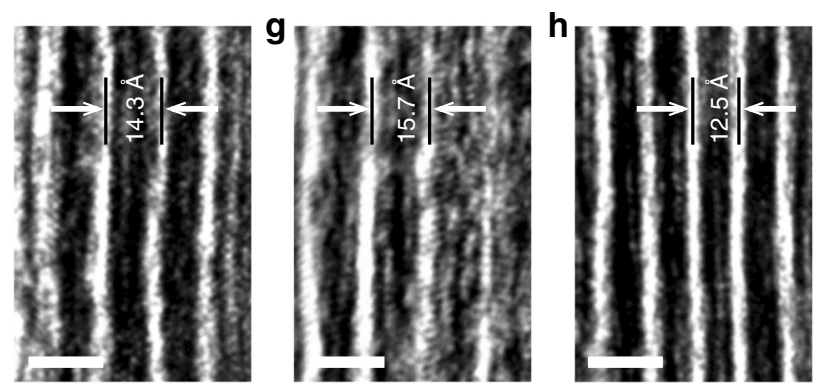

Fig. 5 Hybrid transition metal dichalcogenide (TMD)-nanoscrolls (NSs). a Schematic showing the cross section and sidewalls of hybrid TMD-NSs. b-h HR-TEM images of sidewalls of $\mathrm{MoS}_{2}-\mathrm{NSs}$ hybridized with AuNPs (b), GO (c), pentacene (d), CuPc (e), PDPP3T (f), DNA (g) and polypeptide (h). AuNPs approximately $5 \mathrm{~nm}$ in diameter were clearly observed between the walls; the GO flakes were also distinguishable, as shown in c. Lattice expansion was displayed in all hybrid NSs above. The bright white walls represent the $\mathrm{MoS}_{2}$ layers in (c-h) (scale bars, $10 \mathrm{~nm}$ in $\mathbf{b}$ and $2 \mathrm{~nm}$ in $\mathbf{c}-\mathbf{h}$ )

\section{Discussion}

In summary, we have explored a simple method for producing high-quality, tightly scrolled TMD-NSs from CVD-grown monolayer TMD flakes with the assistance of one drop of an ethanol solution. An obvious PL was shown in TMD-NSs, with a red shift in the direct excitonic transitions. The electron mobility of the $\mathrm{MoS}_{2}$-NSs was approximately 30-fold greater than that of the monolayer $\mathrm{MoS}_{2}$ flakes. This scrolling strategy will be a candidate for increasing mobility of other 2D materials. Owing to their unique self-encapsulated structures, these TMD-NSs show stable optical and electronic properties in different atmospheres. The HR-TEM results show the internal open topological structure of the TMD-NSs, which serve as effective carriers for accommodating external substance of different sizes in their tunable van der Waals gaps. The hybridization with other functional materials will confer TMD-NSs with additional properties and functions attractive for potential application.

\section{Methods}

CVD growth of TMD monolayers. TMD monolayers were grown by an atmospheric pressure CVD. The growth substrates, including $\mathrm{SiO}_{2} / \mathrm{Si}_{3}, \mathrm{Si}_{3} \mathrm{~N}_{4}$ and sapphire, were cleaned in acetone, isopropanol, and deionized water and then dried under high-purity nitrogen flow.
For $\mathrm{MoS}_{2}$ monolayers, the growth substrate was placed face-down above a ceramic boat containing $\mathrm{MoO}_{3}$ powder (Sigma Aldrich, 99.998\%, $20 \mathrm{mg}$ ). The ceramic boat was then loaded in the heating zone center of the furnace tube, where another boat containing $80 \mathrm{mg}$ of sulfur (Alfa Aesar, 99.999\%) was located upstream. The tube was first purged with ultrahigh-purity argon for $10 \mathrm{~min}$ at a flow rate of $200 \mathrm{sccm}$. Then, the furnace was heated from room temperature to 500 ${ }^{\circ} \mathrm{C}$ in $12 \mathrm{~min}, 500-720^{\circ} \mathrm{C}$ in another $20 \mathrm{~min}$, and then stayed at $720^{\circ} \mathrm{C}$ for $5 \mathrm{~min}$. The sulfur was heated to $130^{\circ} \mathrm{C}$ with a separate heat setup as the furnace reached $720^{\circ} \mathrm{C}$. Finally, the temperature was cooled from 720 to $570{ }^{\circ} \mathrm{C}$ in $20 \mathrm{~min}$ before opening the furnace for rapid cooling. Argon as carrier gas was maintained at a flow rate of $10 \mathrm{sccm}$ throughout the growth process.

The growth of $\mathrm{WS}_{2}$ monolayers was similar to that of $\mathrm{MoS}_{2}$, except that $\mathrm{WO}_{3}$ (Alfa Aesar, 99.998\%, $10 \mathrm{mg}$ ) was used as one of the precursors and the highest growth temperature was set at $825^{\circ} \mathrm{C}$, with sulfur at $150^{\circ} \mathrm{C}$.

$\mathrm{WSe}_{2}$ monolayers were grown on substrates with $\mathrm{WO}_{3}(10 \mathrm{mg})$ and selenium powders (Alfa Aesar, $99.999+\%, 1-5 \mathrm{~mm}$ ) as precursors. The growth recipe was as follows: ramp the furnace from room temperature to $825^{\circ} \mathrm{C}$ in $20 \mathrm{~min}$, sit $20 \mathrm{~min}$ at $825^{\circ} \mathrm{C}$, cool to $600^{\circ} \mathrm{C}$ in $20 \mathrm{~min}$, and open the furnace for rapid cooling. The selenium powders were heated to $300^{\circ} \mathrm{C}$ when the furnace temperature rose to 825 ${ }^{\circ} \mathrm{C}$ and were maintained at this temperature for $40 \mathrm{~min} .1 .5 \mathrm{sccm}$ hydrogen and $23.5 \mathrm{sccm}$ argon were used during the growth process.

$\mathrm{MoSe}_{2}$ monolayers were grown via a procedure similar to that used for $\mathrm{WSe}_{2}$ monolayers, except that the source precursors were $\mathrm{MoO}_{3}$ and selenium, and the selenium was kept at $400^{\circ} \mathrm{C}$ during the growth process.

Fabrication of TMD-NSs. TMD-NSs were fabricated by dropping an ethanol aqueous solution onto the CVD-grown TMD monolayers and allowing it to dry naturally. Rod-like NSs were then obtained on the substrate. The concentration of the ethanol aqueous solution ranged from 5:1 to 1:1 (volume ratio of ethanol: water), with an optimum ratio of 2:1.

MoS $_{\mathbf{2}}$-NSs arrays. A $\mathrm{MoS}_{2}$ polycrystalline film was first patterned into parallel ribbons with defined width and direction using a FIB. A drop of pure ethanol was dropped on the patterned film, and then a coverslip was put on top of it. A thin liquid layer of pure ethanol can be formed between the film and the coverslip. An ethanol solution (1:1) was then dropped on the edge of the coverslip. Drove by the concentration gradient, the water in the solution will slowly infiltrate into the pure ethanol under the coverslip in the gradient direction (as shown in Supplementary Fig. 8). The diffusion rate can be adjusted by the concentration of the ethanol solution. In this way one side of a ribbon was first controlled to scroll to produce a long NS. The long NS array was then fabricated and further arranged into periodic arrays with a controlled length by FIB etching a second time.

Hybrid MoS $_{2}$-NSs fabrication. Pentacene/MoS - NSs: a 1-nm-thick layer of pentacene was deposited on the $\mathrm{MoS}_{2}$ monolayers on a $\mathrm{SiO}_{2} / \mathrm{Si}$ substrate by vacuum thermal evaporation, followed by immersion into a modulated ethanol solution (5:1). After $10 \mathrm{~min}$, the substrate was picked up and allowed to dry naturally. The pentacene/MoS 2 -NSs were obtained. The $\mathrm{CuPc} / \mathrm{MoS}_{2}-\mathrm{NSs}$ were prepared in the same way.

PDPP3T/MoS 2 -NSs: A PDPP3T solution was prepared by dissolving $2 \mathrm{mg}$ of PDPP3T powder in $1 \mathrm{ml}$ of toluene. After stirring overnight, the PDPP3T solution was spin-coated onto the $\mathrm{MoS}_{2}$ monolayers at a speed of $5000 \mathrm{rpm}$. After annealing at $70^{\circ} \mathrm{C}$ for $20 \mathrm{~min}$, the PDPP3T-coated $\mathrm{MoS}_{2}$ monolayers were then dipped into an ethanol solution (5:1). After $10 \mathrm{~min}$, the $\mathrm{MoS}_{2}$ monolayers were picked up and dried naturally.

$\mathrm{GO} / \mathrm{MoS}_{2}$-NSs: This procedure is similar to that used for PDPP3T/MoS 2 -NSs. Freshly exfoliated GO sheets were dispersed in $n$-hexane and then spin-coated onto $\mathrm{MoS}_{2}$ monolayers. After dropping an ethanol solution (2:1) onto the samples, the $\mathrm{GO} / \mathrm{MoS}_{2}$-NSs were obtained.

$\mathrm{Au} / \mathrm{MoS}_{2}$-NSs: $\mathrm{MoS}_{2}$ monolayers were dipped into an ethanol solution (2:1) containing AuNPs. Ten minutes later, the $\mathrm{MoS}_{2}$ monolayers were picked up and dried naturally. Similar procedures were used for producing both DNA/MoS ${ }_{2}$ and polypetide/ $\mathrm{MoS}_{2}$-NSs.

MoS 2 -NS-based FET. Freshly made monolayer $\mathrm{MoS}_{2}$ flakes on a $\mathrm{SiO}_{2} / \mathrm{Si}$ substrate were kept in nitrogen for $48 \mathrm{~h}$. Then the $\mathrm{MoS}_{2}$ flakes were scrolled into NSs with one drop of ethanol solution (2:1) in nitrogen atmosphere. Bottom-gated transistors based on these $\mathrm{MoS}_{2}$-NSs were fabricated on the $\mathrm{SiO}_{2} / \mathrm{Si}$ substrate without additional transfer. $100 \mathrm{~nm}$ of Au was deposited on the top of $\mathrm{MoS}_{2}$-NSs as source and drain electrodes with a shadow mask by vacuum thermal evaporation at a rate of about $0.3 \AA \mathrm{s}^{-1}$. The electrical measurements were conducted under a nitrogen atmosphere and ambient condition, respectively.

Characterization. Raman and PL measurements were performed with a $532 \mathrm{~nm}$ laser under ambient conditions (inVia-Reflex). All optical images and the video were captured with a Nikon Eclipse LV100D. The fluorescence microscope characterization was performed using a fluorescence microscope equipped with a mercury lamp as the excitation light source. Large-area $\mathrm{MoS}_{2}$ films and long $\mathrm{MoS}_{2}$-NSs were etched via FIB-SEM (FEI, Helios Nanolab G3 CX). SEM 
(Hitachi S-4800) and HR-TEM (JEOL JEM-2100F) were employed to image the samples. Current-voltage curves were obtained by a three-probe station under a nitrogen atmosphere and ambient conditions (Keithley 4200 SCS).

Data availability. The data that support the findings of this study are available from the corresponding author on reasonable request.

Received: 24 August 2017 Accepted: 8 March 2018

Published online: 03 April 2018

\section{References}

1. Wang, Q. H., Kalantar-Zadeh, K., Kis, A., Coleman, J. N. \& Strano, M. S. Electronics and optoelectronics of two-dimensional transition metal dichalcogenides. Nat. Nanotechnol. 7, 699-712 (2012).

2. Chhowalla, M. et al. The chemistry of two-dimensional layered transition metal dichalcogenide nanosheets. Nat. Chem. 5, 263-275 (2013).

3. Kang, K. et al. High-mobility three-atom-thick semiconducting films with wafer-scale homogeneity. Nature 520, 656-660 (2015).

4. Chen, J. et al. Chemical vapor deposition of large-size monolayer $\mathrm{MoSe}_{2}$ crystals on molten glass. J. Am. Chem. Soc. 139, 1073-1076 (2017).

5. Gong, Y. et al. Vertical and in-plane heterostructures from $\mathrm{WS}_{2} / \mathrm{MoS}_{2}$ monolayers. Nat. Mater. 13, 1135-1142 (2014).

6. Duan, X. et al. Lateral epitaxial growth of two-dimensional layered semiconductor heterojunctions. Nat. Nanotechnol. 9, 1024-1030 (2014).

7. $\mathrm{Li}, \mathrm{M}$. Y. et al. Epitaxial growth of a monolayer $\mathrm{WSe}_{2}-\mathrm{MoS}_{2}$ lateral pn junction with an atomically sharp interface. Science 349, 524-528 (2015).

8. $\mathrm{Li}, \mathrm{X}$. et al. Two-dimensional GaSe/MoSe 2 misfit bilayer heterojunctions by van der Waals epitaxy. Sci. Adv. 2, e1501882 (2016).

9. Pan, H., Feng, Y. \& Lin, J. Ab initio study of electronic and optical properties of multiwall carbon nanotube structures made up of a single rolled-up graphite sheet. Phys. Rev. B 72, 085415 (2005).

10. Chen, Y., Lu, J. \& Gao, Z. Structural and electronic study of nanoscrolls rolled up by a single graphene sheet. J. Phys. Chem. C. 111, 1625-1630 (2007).

11. Chang, C. \& Ortix, C. Theoretical prediction of a giant anisotropic magnetoresistance in carbon nanoscrolls. Nano Lett. 17, 3076-3080 (2017)

12. Liu, Z., Gao, J., Zhang, G., Cheng, Y. \& Zhang, Y. From two-dimensional nano-sheets to roll-up structures: expanding the family of nanoscroll. Nanotechnology 28, 385704 (2017).

13. Zheng, J. et al. Production of high-quality carbon nanoscrolls with microwave spark assistance in liquid nitrogen. Adv. Mater. 23, 2460-2463 (2011).

14. Zheng, B., Xu, Z. \& Gao, C. Mass production of graphene nanoscrolls and their application in high rate performance supercapacitors. Nanoscale $\mathbf{8}$, 1413-1420 (2016).

15. Berman, D., Deshmukh, S. A., Sankaranarayanan, S. K. R. S., Erdemir, A. \& Sumant, A. V. Macroscale superlubricity enabled by graphene nanoscroll formation. Science 348, 1118-1122 (2015).

16. Xie, X. et al. Controlled fabrication of high-quality carbon nanoscrolls from monolayer graphene. Nano Lett. 9, 2565-2570 (2009).

17. Mpourmpakis, G., Tylianakis, E. \& Froudakis, G. E. Carbon nanoscrolls: a promising material for hydrogen storage. Nano Lett. 7, 1893-1897 (2007).

18. Shi, X., Cheng, Y., Pugno, N. M. \& Gao, H. A translational nanoactuator based on carbon nanoscrolls on substrates. Appl. Phys. Lett. 96, 053115 (2010).

19. Chen, X., Boulos, R. A., Dobson, J. F. \& Raston, C. L. Shear induced formation of carbon and boron nitride nano-scrolls. Nanoscale 5, 498-502 (2013).

20. Thangasamy, P. \& Sathish, M. Rapid, one-pot synthesis of luminescent $\mathrm{MoS}_{2}$ nanoscrolls using supercritical fluid processing. J. Mater. Chem. C. 4 1165-1169 (2016).

21. Meng, J. et al. Rolling up a monolayer $\mathrm{MoS}_{2}$ sheet. Small 12, 3770-3774 (2016).

22. Gutiérrez, H. R. et al. Extraordinary room-temperature photoluminescence in triangular $\mathrm{WS}_{2}$ monolayers. Nano Lett. 13, 3447-3454 (2012).

23. Van Der Zande, A. M. et al. Grains and grain boundaries in highly crystalline monolayer molybdenum disulfide. Nat. Mater. 12, 554-561 (2013).

24. Chen, J. et al. Chemical vapor deposition of large-sized hexagonal $\mathrm{WSe}_{2}$ crystals on dielectric substrates. Adv. Mater. 27, 6722-6727 (2015).

25. Lee, C. et al. Anomalous lattice vibrations of single-and few-layer $\mathrm{MoS}_{2}$. ACS Nano 4, 2695-2700 (2010).

26. $\mathrm{Li}, \mathrm{H}$. et al. From bulk to monolayer $\mathrm{MoS}_{2}$ : evolution of Raman scattering. Adv. Funct. Mater. 22, 1385-1390 (2012).
27. Wieting, T. J. \& Verble, J. L. Interlayer bonding and the lattice vibrations of $\beta$-GaSe. Phys. Rev. B 5, 1473-1479 (1972).

28. Rice, C. et al. Raman-scattering measurements and first-principles calculations of strain-induced phonon shifts in monolayer $\mathrm{MoS}_{2}$. Phys. Rev. B 87, 081307 (2013).

29. Mak, K. F., Lee, C., Hone, J., Shan, J. \& Heinz, T. F. Atomically thin $\mathrm{MoS}_{2}$ : a new direct-gap semiconductor. Phys. Rev. Lett. 105, 136805 (2010).

30. Britnell, L. et al. Strong light-matter interactions in heterostructures of atomically thin films. Science 340, 1311-1314 (2013).

31. Lopez-Sanchez, O., Lembke, D., Kayci, M., Radenovic, A. \& Kis, A. Ultrasensitive photodetectors based on monolayer $\mathrm{MoS}_{2}$. Nat. Nanotechnol. 8, 497-501 (2013).

32. He, K., Poole, C., Mak, K. F. \& Shan, J. Experimental demonstration of continuous electronic structure tuning via strain in atomically thin $\mathrm{MoS}_{2}$. Nano Lett. 13, 2931-2936 (2013).

33. Seifert, G., Terrones, H., Terrones, M., Jungnickel, G. \& Frauenheim, T. Structure and electronic properties of $\mathrm{MoS}_{2}$ nanotubes. Phys. Rev. Lett. 85, 146 (2000).

34. Najmaei, S. et al. Vapour phase growth and grain boundary structure of molybdenum disulphide atomic layers. Nat. Mater. 12, 754-759 (2013).

35. Fu, D. et al. Molecular beam epitaxy of highly crystalline monolayer molybdenum disulfide on hexagonal boron nitride. J. Am. Chem. Soc. 139 9392-9400 (2017)

36. Kim, S. et al. High-mobility and low-power thin-film transistors based on multilayer $\mathrm{MoS}_{2}$ crystals. Nat. Commun. 3, 1011 (2012).

37. Gao, L. et al. Face-to-face transfer of wafer-scale graphene films. Nature $\mathbf{5 0 5}$, 190-194 (2014).

\section{Acknowledgements}

We thank Bo Guan and Xiang Li for the assistance with FIB-SEM. We thank Bo Guan for the assistance in SAED analysis. This work was supported by the Strategic Priority Research Programme of the National Natural Science Foundation (21573253), and the Chinese Academy of Sciences (XDB12010000).

\section{Author contributions}

X.C. performed the experiments, analyzed the data, and wrote the paper. J.Z. designed the work, performed the experiments, conceptualized the work, and analyzed the data. Z.K., D.H., Y.H., H.S., C.D., and D.Z. performed some characterization experiments and analyzed data. E.G. and Z.X. performed the theoretical analysis.

\section{Additional information}

Supplementary Information accompanies this paper at https://doi.org/10.1038/s41467018-03752-5.

Competing interests: The authors declare no competing interests.

Reprints and permission information is available online at http://npg.nature.com/ reprintsandpermissions/

Publisher's note: Springer Nature remains neutral with regard to jurisdictional claims in published maps and institutional affiliations.

Open Access This article is licensed under a Creative Commons Attribution 4.0 International License, which permits use, sharing, adaptation, distribution and reproduction in any medium or format, as long as you give appropriate credit to the original author(s) and the source, provide a link to the Creative Commons license, and indicate if changes were made. The images or other third party material in this article are included in the article's Creative Commons license, unless indicated otherwise in a credit line to the material. If material is not included in the article's Creative Commons license and your intended use is not permitted by statutory regulation or exceeds the permitted use, you will need to obtain permission directly from the copyright holder. To view a copy of this license, visit http://creativecommons.org/ licenses/by/4.0/

(c) The Author(s) 2018 